\title{
Prominent Systolic Coronary Flow in a Coronary Artery Fistula with a Giant Aneurysma
}

\author{
Tomoyuki HorI, ${ }^{1} \mathrm{MD}$, Taku MATSUBARA, ${ }^{1} \mathrm{MD}$, Iwao NAKAGAwA, ${ }^{1} \mathrm{MD}$, \\ Shunsuke IMAI, ${ }^{1}$ MD, Kazuyuki OzAKI, ${ }^{1}$ MD, Katsuharu HATADA, ${ }^{1}$ MD, \\ Keiichi TsuchidA, ${ }^{1}$ MD, Hiroshi WATANABE, ${ }^{1}$ MD, Masaya KitAmura, ${ }^{1}$ MD, \\ Jun-ichi HAYASHI, ${ }^{2} \mathrm{MD}$, and Yoshifusa AIZAWA, ${ }^{1} \mathrm{MD}$
}

\begin{abstract}
SUMMARY
A 68-year-old Japanese woman was admitted to hospital because of chest oppression during exertion. Coronary angiography showed a coronary artery fistula with a giant aneurysm, which originated from both the left anterior descending (LAD) and right coronary arteries. We investigated coronary blood flow velocity using the Doppler guide wire technique. The coronary flow pattern showed a very prominent systolic component, whereas the diastolic flow components were nearly normal before the operation at the LAD site proximal to the coronary artery fistula. This pattern returned to normal after the operation. This report describes the relationship between the coronary steal phenomenon and coronary flow dynamics investigated directly using the Doppler guidewire technique. (Jpn Heart J 2001; 42: 525-531)
\end{abstract}

Key words: Coronary artery fistula, Coronary steal, Doppler guide wire

CORONARY artery fistulas originating from both coronary arteries with a giant saccular aneurysm are very rare. ${ }^{1-3)}$ Recently, with the widespread use of coronary angiography, they have been recognized more often. Although young patients with coronary artery fistulas frequently remain asymptomatic, about half of these patients develop cardiovascular symptoms, such as angina, dyspnea on exertion, and fatigue with aging. ${ }^{4}$ Chest pain associated with this anomaly is explained by the coronary steal phenomenon, based on the relief of angina after ligation of the coronary artery fistula. ${ }^{4,5)}$ However, little information is available concerning the relationship between the coronary steal phenomenon and coronary blood flow. Therefore, we investigated coronary blood flow velocity dynamics, before and after surgery, using the Doppler guide wire technique in a patient with a coronary artery fistula.

From ${ }^{1}$ the First Department of Internal Medicine, ${ }^{2}$ Second Department of Surgery, Niigata University School of Medicine, Niigata, Japan.

Address for correspondence: Tomoyuki Hori, MD, First Department of Internal Medicine, Niigata University School of Medicine, 1-754 Asahi-machi-dori, Niigata, Niigata 951-8510, Japan.

Received for publication February 15, 2001.

Revised and accepted March 8, 2001. 


\section{Case Report}

A 68-year-old Japanese woman was admitted for investigation of a chest Xray abnormality and assessment of chest oppression. A high-pitched, continuous Levine 2/6 murmur was heard in the third intercostal space at the left sternal border. Chest X-ray examination showed a bulge at the left cardiac border with linear calcification. An electrocardiogram showed sinus rhythm and no significant ST-T wave changes. Transthoracic echocardiography demonstrated a mass, with a maximum diameter of $5 \mathrm{~cm}$, located in front of the main pulmonary artery (PA). Thallium-201 scintigraphy revealed no perfusion defect, and computed tomography showed a round mass with a maximum diameter of $5 \mathrm{~cm}$. Cardiac catheterization showed normal pressure values in the right and left sides of the heart and no left-right shunt. Coronary angiography revealed a coronary artery fistula with a giant aneurysm, which originated from both the left anterior descending (LAD) and right coronary arteries (Figure 1). This giant aneurysm received contrast material from the LAD. The contrast material in this aneurysm cleared slowly and drained into the main PA. The fistula arising from the right coronary artery also drained into the main PA. We analyzed coronary blood flow velocity dynamics before and after surgery using a Doppler guide wire (Flowire, Cardiometrics, Mountain View, CA). After diagnostic angiography, the Doppler guide wire was passed through an angioplasty $\mathrm{Y}$-connector attached to a $6 \mathrm{~F}$ diagnostic catheter, and then advanced into the LAD. Doppler velocities were recorded at several points from the proximal to the distal part of the LAD. Figure 2 shows the Doppler velocities at sites proximal and distal to the coronary fistula before the operation. The proximal average peak velocity was $30 \mathrm{~cm} / \mathrm{s}$ and the distal $29 \mathrm{~cm} / \mathrm{s}$. In addition, the diasto/lic-to-systolic velocity ratio (DSVR) was 0.4 and 1.2, respectively. Noticeably, in the LAD proximal to the coronary artery fistula, the coronary flow velocity pattern showed a predominant systolic pattern with higher peak systolic velocities and flow velocity integrals. On the other hand, in the LAD distal to the fistula, the pattern was almost normal, showing a predominant diastolic pattern with higher peak diastolic velocities and flow velocity integrals. Coronary blood flow was estimated from coronary blood flow velocity and diameter measurements using the formula $\pi \times$ averaged peak velocity $\mathrm{x} 0.125 \mathrm{x}$ diameter. $^{2,6)}$ Coronary flow was $211 \mathrm{ml} / \mathrm{min}$ and $64 \mathrm{ml} / \mathrm{min}$ in the LAD proximal and distal to the fistula, respectively. Considering the possibility of sudden rupture with catastrophic results, surgical repair was undertaken. The heart was exposed through a median sternotomy. A giant coronary aneurysm was located on the left side of the PA and was connected to be bilateral coronary artery-pulmonary artery fistulae. Anomalous vessels originated from the LAD and the right coronary artery. The anomalous vessel originated from the proximal side of the LAD and 

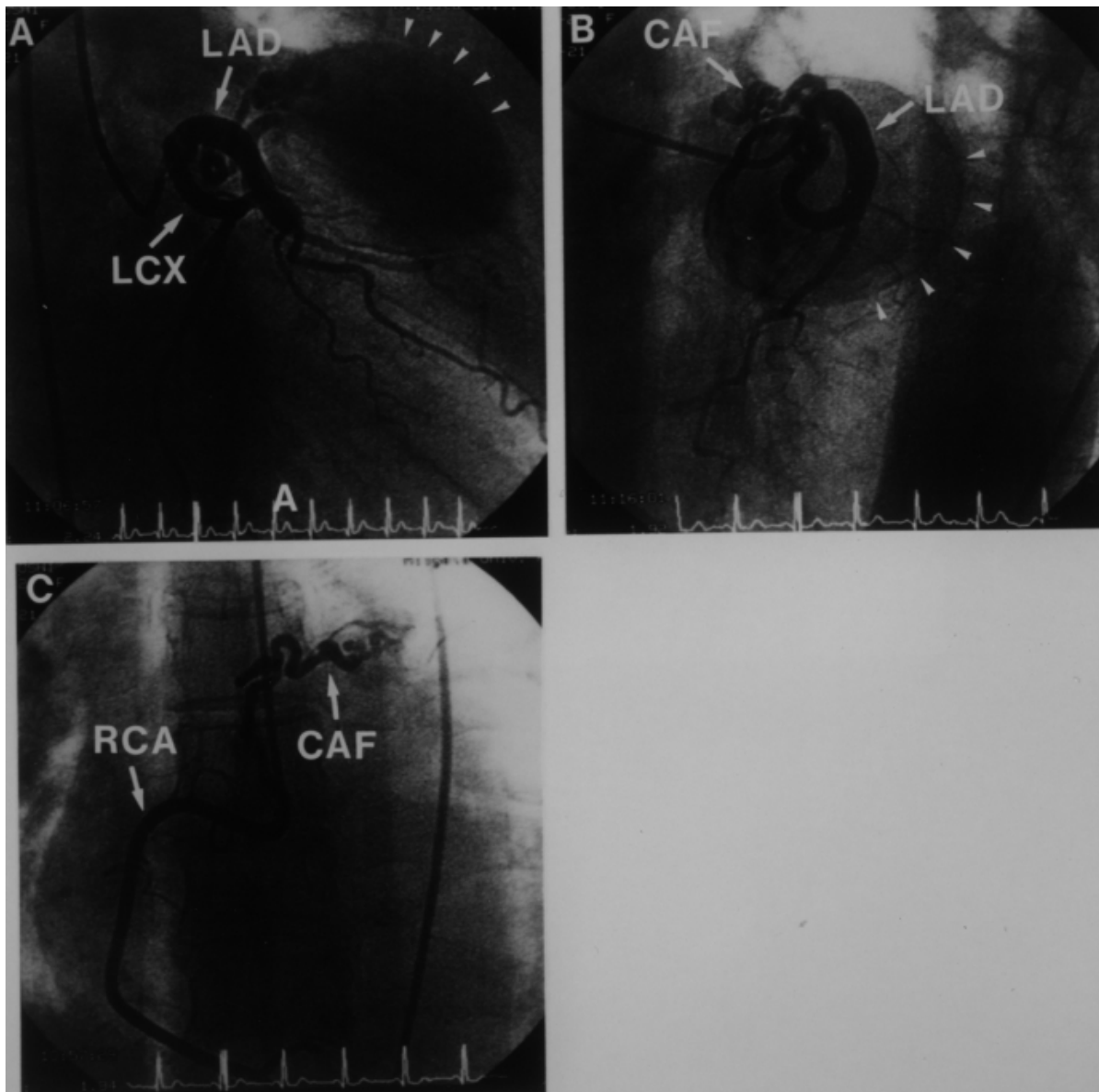

Figure 1. Coronary artery fistula, originating from both coronary arteries, with a giant saccular aneurysm (arrowheads). A: Left coronary angiogram in right anterior oblique (RAO) view; B: Left coronary angiogram in left anterior oblique (LAO) view; $\mathbf{C}$ : Right coronary angiogram in posterior anterior view. $\mathrm{LAD}=\mathrm{left}$ anterior descending artery; $\mathrm{LCX}=\mathrm{left}$ circumflex artery; $\mathrm{CAF}=$ coronary artery fistula.

branched into two vessels. One drained into the main PA via a network of vessels which originated from the right coronary artery, and the other was directly connected to the giant aneurysm and finally drained into the main PA. Ligation of the coronary artery fistula, closure of the orifice of the artery draining to the PA and aneurysmorrhaphy were performed for this giant saccular aneurysm. After the operation, we measured coronary flow velocity again. A Doppler guide wire was placed into the same arterial site of the LAD. Figure 3 shows the coronary flow velocities after the operation. The proximal and distal average peak velocities 

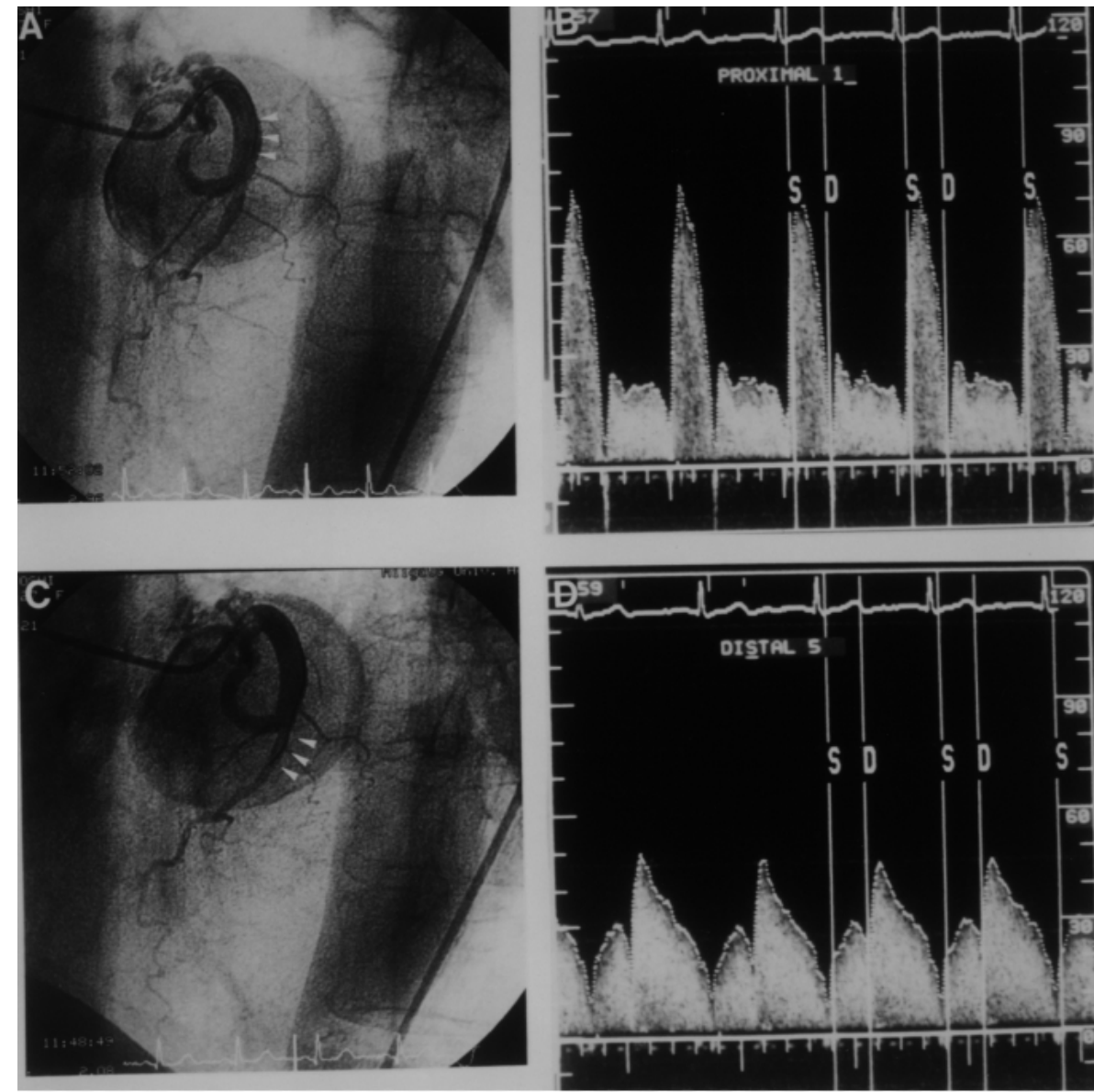

Figure 2. Before surgery. A: The Doppler guidewire is placed at the LAD site proximal to the coronary artery fistula (arrowheads). B: Doppler flow velocity at the LAD site proximal to the coronary artery fistula. C: The Doppler guidewire is placed at the LAD site distal to the coronary artery fistula (arrowheads). D: Doppler flow velocity at the LAD site distal to the coronary artery fistula.

were $10 \mathrm{~cm} / \mathrm{s}$ and $23 \mathrm{~cm} / \mathrm{s}$, respectively. The DSVR was 1.3 and 1.3 , respectively. At the LAD proximal to the coronary artery fistula, the coronary flow velocity pattern recovered to nearly normal. Coronary flow was $72 \mathrm{ml} / \mathrm{min}$ and $53 \mathrm{ml} / \mathrm{min}$ at the LAD sites proximal and distal to the fistula, respectively. The postoperative course was uncomplicated and the patient has remained asymptomatic. 

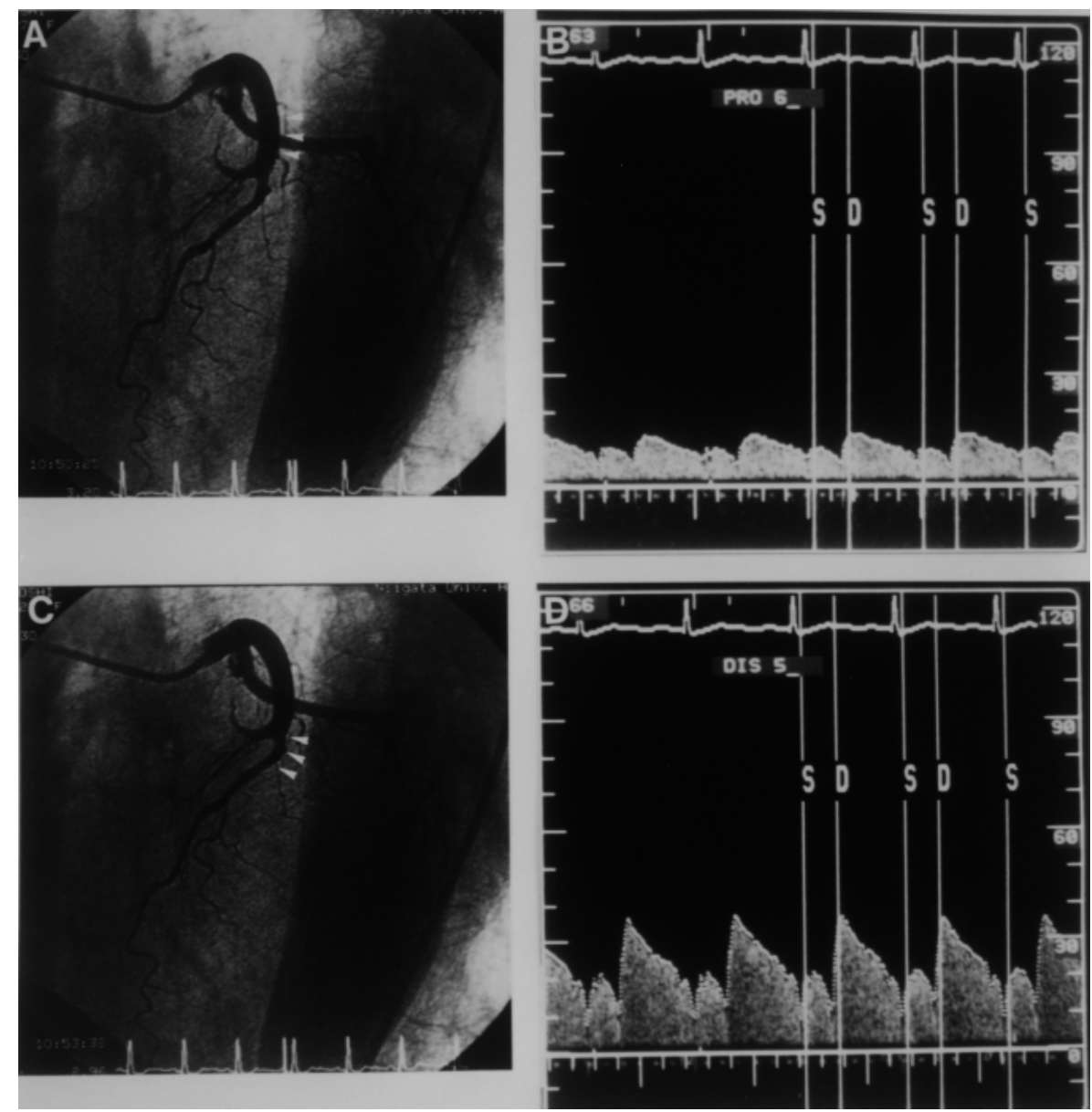

Figure 3. After surgery. A: The Doppler guidewire is placed at the LAD site proximal to the coronary artery fistula (arrowheads). B: Doppler flow velocity at the LAD site proximal to the coronary artery fistula. C: The Doppler guidewire is placed at the LAD site distal to the coronary artery fistula (arrowheads). D: Doppler flow velocity at the LAD site distal to the coronary artery fistula.

\section{DISCUSSION}

We examined the coronary blood flow dynamics at sites proximal and distal to the coronary fistula and demonstrated directly a relationship between the coronary steal phenomenon and the coronary blood flow velocity dynamics. Before surgery, coronary blood flow had decreased to about $150 \mathrm{ml} / \mathrm{min}$ behind the coronary artery-pulmonary artery fistula with a giant saccular aneurysm. Ikeda, et $a l^{7)}$ using an electromagnetic flow probe during the operation, reported that the right and left coronary shunt flows were both $100 \mathrm{ml} / \mathrm{min}$. Okeie, et al ${ }^{8)}$ measured the coronary flow at the site distal to the fistula during atrial pacing using a 
Doppler flow wire before and after surgery. Their study showed that blood flow in the LAD decreased during rapid atrial pacing before the operation, compared with that after the operation. However, they did not examine coronary blood flow and its pattern in front of and behind the coronary artery fistula.

In patients with significant coronary stenosis, diastolic flow components are significantly reduced and systolic flow components are less affected. Briefly, coronary stenosis reduces the normal diastolic predominance of the phasic pattern, and the DSVR falls. After angioplasty to the coronary stenosis, the diastolic flow and DSVR increase significantly, whereas systolic flow does so to a lesser extent. ${ }^{9)}$ In our patient, the coronary flow pattern showed a very prominent systolic component, and the diastolic flow components were nearly normal before surgery. This pattern was clearly different from that of significant coronary stenosis. Although there are limited data on fistula flow in patients with a coronary artery-pulmonary artery fistula, Reitze, et $\mathrm{al}^{10)}$ reported that a continuous systolic and diastolic flow pattern was evident in the proximal coronary artery and fistula in an experimental model of coronary artery fistula. However, Okeie, et al ${ }^{8)}$ using the Doppler wire, reported that fistula flow was observed mainly in systole in a patient with aneurysmal formation in the large coronary artery fistula. Given the difference in the fistula flow pattern with and without the giant aneurysm, we considered that the systolic acceleration of coronary flow velocity resulted from drainage into the main PA via the aneurysm, which acts as a low-pressure chamber.

There have been several reports in which the coronary steal phenomenon has been documented by stress thallium-201 scintigraphy. ${ }^{11,12)}$ However, most of these data were obtained from patients with coronary artery-left ventricular fistulae, and reports documenting coronary artery-pulmonary artery fistula have been scarce. Okeie, et $a l^{13)}$ performed stress thallium-201 scintigraphy in 7 patients with coronary artery-pulmonary artery fistula to elucidate the relationship with myocardial ischemia. Their results showed typical findings of reversible myocardial ischemia due to the coronary steal phenomenon in only 2 of 7 patients. Therefore, most patients with coronary artery-pulmonary artery fistula did not always show typical signs of myocardial ischemia due to the coronary steal phenomenon. In our patient, reversible myocardial ischemia was not detected by exercise-stress thallium-201 scintigraphy. For this reason, we speculated that the diastolic coronary flow around the coronary fistula might be less affected than the systolic coronary flow by coronary steal due to the coronary artery fistula.

This report has described the relationship between the coronary steal phenomenon and coronary flow dynamics investigated directly using the Doppler guidewire technique. 


\section{REFERENCES}

1. Takahashi M, Seguchi H, Fujikawa H, et al. Multicystic aneurysmal dilatation of bilateral coronary artery fistula. Cathet Cardiovasc Diagn 1994; 31: 290-2.

2. Okita Y, Miki S, Kusuhara K, et al. Aneurysm of coronary arteriovenous fistula presenting as a calcified mediastinal mass. Ann Thorac Surg 1992; 54: 771-3.

3. Egashira K, Otsubo H, Furuyama T, Tomoike H, Takeshita A, Nakamura M: Multiple coronary-to-pulmonary artery fistulas with progressive aneurysmal dilatation. Am Heart J 1984; 108: 1038-40.

4. Liberthson RR, Sagar K, Berkoben JP, Weintraub RM, Levene FH: Congenital coronary arteriovenous fistula: Report of 13 patients, review of the literature and delineation of management. Circulation 1979; 59: 849-54.

5. Rowe GG: Inequality of myocardial perfusion in coronary disease ("coronary steal"). Circulation 1970; 42: 251-4.

6. Doucette JW, Corl D, Payne HM, et al. Validation of a Doppler guide wire for intravascular measurement of coronary artery flow velocity. Circulation 1992; 85: 1899-911.

7. Ikeda M, Ishikawa N, Takahashi M, et al. A successful surgical treatment of coronary to pulmonary artery fistula with coronary steal phenomenon due to large volume of shunt: A case report. Jpn J Thorac Surg 1997; 50: 230-3.

8. Okeie K, Horita Y, Shimazu M, Mabuchi H. A quantitative evaluation of coronary steal phenomenon in coronary artery - pulmonary artery fistula: Case report. J Cardiol 1995; 26: 305-12.

9. Segal J, Kern MJ, Scott NA, et al. Alterations of phasic coronary artery flow velocity in humans during percutaneous coronary angioplasty. J Am Coll Cardiol 1992; 20: 276-86.

10. Reitz BA, Harrison LH, Michaelis LL. Experimental coronary artery fistula. J Thorac Cardiovasc Surg 1975; 69: 278-82.

11. Origuchi H, Shimokawa H, Sugihara M, Sagara T, Kikuchi Y. Demonstration of exercise-induced myocardial ischemia in a case of multiple coronary arterioventricular fistulae. Am Heart J 1986; 112: 1096-9.

12. Oshiro K, Shimabukuro M, Nakada, Y et al. Multiple coronary LV fistulae: Demonstration of coronary steal phenomenon by stress thallium scintigraphy and exercise hemodynamics. Am Heart J 1990; 120: 217-9.

13. Okeie K, Mizuno S, Nitta Y, Matsubara T. Clinical features of congenital coronary artery fistula in adult population: Relationship between the form of bifurcation and coronary steal phenomenon. Shinzo 1994; 26: 102937. 